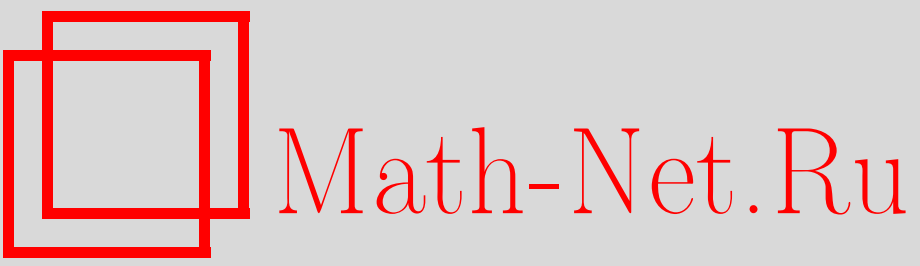

С. Д. Гриффитс, Р. Гримшоу, К. Р. Хуснутдинова, Влияние модуляционной неустойчивости на обмен энергией в связанных уравнениях синус-Гордон, ТМФ, 2003, том 137, номер 1, 108-120

DOI: https://doi.org/10.4213/tmf249

Использование Общероссийского математического портала Math-Net.Ru подразумевает, что вы прочитали и согласны с пользовательским соглашением

http://www. mathnet.ru/rus/agreement

Параметры загрузки:

IP: 3.81 .55 .215

26 апреля 2023 г., 03:37:55 
ТЕОРЕТИЧЕСКАЯ

И МАТЕМАТИЧЕСКАЯ

ФИЗИКА

Том 137, № 1

октябрь, 2003

(C) 2003 г.

С. Д. Гриффитс ${ }^{*}$ Р. Г. Дж. Гримшоу*,

\author{
К.Р. Хуснутдинова ${ }^{*} \dagger$
}

\title{
ВЛИЯНИЕ МОДУЛЯЦИОННОЙ НЕУСТОЙЧИВОСТИ НА ОБМЕН ЭНЕРГИЕЙ В СВЯЗАННЫХ УРАВНЕНИЯХ СИНУС-ГОРДОН
}

Рассматривается двухкомпонентная система связанных уравнений типа синус-Гордон. Частные решения этой системы задают обобщение на непрерывный случай процесса с периодическим обменом энергией, имеющего место в системе связанных маятников. Слабонелинейные решения, которые описывают процесс периодического обмена энергией, происходящего между волнами, распространяющимися в двух компонентах, задаются в зависимости от масштаба пространственного изменения амплитуд либо двумя нелокально связанными нелинейными уравнениями Шредингера, в которых члены, отвечающие за перенос масс, различны ввиду различия между групповыми скоростями, либо моделью, оказывающейся бездисперсионной в главном порядке. $\mathrm{C}$ помощью асимптотического анализа и численных экспериментов удается показать, что эффекты дисперсии оказывают существенное влияние на структуру указанных решений и приводят к модуляционной неустойчивости и образованию локализованных структур, но тем не менее сохраняют картину обмена энергией между компонентами системы.

Ключевые слова: связанные уравнения синус-Гордон, амплитудные уравнения, линейные волны, нелинейные волны, обмен энергией в двухкомпонентных системах, модуляционная нестабильность.

\section{1. ВВЕДЕНИЕ}

Система связанных уравнений Клейна-Гордона

$$
u_{t t}-u_{x x}=f_{u}(u, w), \quad w_{t t}-c^{2} w_{x x}=f_{w}(u, w),
$$

где нижние индексы обозначают частные производные, возникает в целом ряде приложений (см., например, [1]-[3]). В этой системе $f(u, w)$ представляет собой потенциальную функцию нелинейной связи, а величина $с$ задает отношение акустических скоростей компонент $u$ и $w$. В простейшем виде уравнения (1) описывают динамику длинных волн

*Department of Mathematical Sciences, Loughborough University, Loughborough, LE11 3TU, UK. E-mail: K.Khusnutdinova@lboro.ac.uk

${ }^{\dagger}$ Постоянное место работы: Институт механики, ул. Карла Маркса 6, Уфа, Россия 
в двух связанных между собой одномерных периодических цепочках частиц, в которых связи между элементами внутри каждой цепочки линейны, а связи между элементами различных цепочек нелинейны.

Система уравнений (1) представляет собой лагранжеву систему с лагранжевой плотностью

$$
L=\frac{1}{2}\left(u_{t}^{2}+w_{t}^{2}-u_{x}^{2}-c^{2} w_{x}^{2}\right)+f(u, w) .
$$

Для любой функции $f(u, w)$ в этой системе присутствуют два закона сохранения - энергии и импульса:

$$
\begin{gathered}
\frac{\partial}{\partial t}\left[\frac{1}{2}\left(u_{t}^{2}+w_{t}^{2}+u_{x}^{2}+c^{2} w_{x}^{2}\right)-f(u, w)\right]-\frac{\partial}{\partial x}\left[u_{t} u_{x}+c^{2} w_{t} w_{x}\right]=0 \\
\frac{\partial}{\partial t}\left[u_{t} u_{x}+w_{t} w_{x}\right]-\frac{\partial}{\partial x}\left[\frac{1}{2}\left(u_{t}^{2}+w_{t}^{2}+u_{x}^{2}+c^{2} w_{x}^{2}\right)+f(u, w)\right]=0
\end{gathered}
$$

Групповая классификация системы уравнений (1) по точечным симметриям была проведена в работе [1] при $c \neq 1, f_{u w}(u, w) \neq 0$. Более обший случай системы $(1)$ при $c=1$ (с произвольными функциями от $u$ и $w$ в правой части) был исследован в работе [4], в которой были найдены примеры уравнений, допускающих симметрии Ли-Беклунда, а потому являющихся (полностью или частично) интегрируемыми. В случае $f_{u w}(u, w)=0$ система уравнений (1) распадается на два независимых уравнения Клейна-Гордона, групповая классификация которых по точечным симметриям была проведена в работе [5] (см. также [6]), а по симметриям Ли-Беклунда - в статье [7].

В случае, когда потенциальная функция имеет вид $f(u, w)=\cos (\delta u-w)-1$, система (1) может быть приведена к системе связанных уравнений синус-Гордон

$$
u_{t t}-u_{x x}=-\delta^{2} \sin (u-w), \quad w_{t t}-c^{2} w_{x x}=\sin (u-w),
$$

где переменная $u$ заменяет собой переменную $\delta u$ в исходной системе (1). Случай связанных уравнений синус-Гордон является обобщением известной модели дислокации Френкеля-Конторовой [8] (см. также работу [9] и приведенную там литературу) и, кроме того, представляет собой модель одномерных нелинейных волновых процессов, протекающих в двухслойном материале с неидеальным интерфейсом [10]. Отметим также, что система уравнений (2) с параметром $c$, близким к единице, была предложена в качестве модели, описывающей открытые состояния молекулы ДНК [2].

Инвариантные решения системы уравнений (2), а также решения, описывающие динамику системы в присутствии дополнительных сдвиговых сил, были исследованы в работе [1]. Пространственно-периодические линейные и нелинейные решения, допускающие обмен энергией между двумя физическими компонентами системы и задающие обобщение на непрерывньй случай процесса обмена энергией в системе связанных маятников [11] (см. также [12]), были исследованы в работе [13]. В настоящей работе исследуется поведение некоторых из найденных частных решений под влиянием малых возмущений. 
Статья имеет следуюшую структуру. В разделе 2 кратко обсуждаются линейные решения, в которых имеет место периодический обмен энергией между волнами, распространяюшимися по двум компонентам. В разделе 3 найдены асимптотические модели, описывающие слабонелинейные решения в двух различных режимах: с наличием или отсутствием дисперсионных членов. В разделе 4 исследуется бездисперсионная асимптотическая модель. Влияние дисперсии исследуется в разделе 5 для случая периодической задачи. При этом используется другая асимптотическая модель. Случай, когда параметр $c$ близок к единице, оказывается привлекательным как с точки зрения наличия аналитического решения в пределе $c=1$, так и с точки зрения потеншиальных приложений получаемых результатов к задачам описания процессов в молекулах ДНК и в двухслойных материалах, и потому этот случай рассматривается отдельно. Результаты численного эксперимента для исходной системы связанных уравнений синус-Гордон (2) обсуждаются и сравниваются с теоретическими предсказаниями в разделе 6 . Краткое заключение содержится в разделе 7 .

\section{2. ОБМЕН ЭНЕРГИЕЙ В ЛИНЕЙНОЙ СИСТЕМЕ}

В случае, когда $|u-w| \ll 1$, связанные уравнения синус-Гордон (2) сводятся к линейной системе. Решения ищутся в виде $(u, w)=(1, \alpha) e^{i(k x-\omega t)}+$ к.с., что приводит к дисперсионному соотношению

$$
\omega_{1,2}^{2}=\frac{1}{2}\left[\nu_{1}^{2}+\nu_{2}^{2} \mp \sqrt{\left(\nu_{1}^{2}-\nu_{2}^{2}\right)^{2}+4 \delta^{2}}\right],
$$

где $\nu_{1}^{2}=\delta^{2}+k^{2}$ и $\nu_{2}^{2}=1+c^{2} k^{2}$. Ввиду симметрии достаточно рассмотреть дисперсионную кривую только в первом квадранте $k>0, \omega>0$. Кривая состоит из двух ветвей $\omega_{1}$ и $\omega_{2}$, типичная форма которых показана на рис. 1 в случаях $c=1$ и $c \neq 1$. Соответствующие значения параметра $\alpha$, задаюшие отношение $w / u$, имеют вид

$$
\alpha_{1,2}=\frac{\nu_{1}^{2}-\omega_{1,2}^{2}}{\delta^{2}}=\frac{1}{\nu_{2}^{2}-\omega_{1,2}^{2}} .
$$

Заметим, что $\alpha_{1}>0$, а $\alpha_{2}<0$. Например, в случае $c=1$ имеют место соотношения $\alpha_{1}=1$ и $\alpha_{2}=-\delta^{-2}$.

Рассмотрим теперь суперпозицию двух линейных волн, выбранных таким образом, что обе движутся слево направо и имеют одно и то же волновое число $k$, но различные частоты $\omega_{1}(k)$ и $\omega_{2}(k)$. Более того, будем предполагать, что одна из компонент, например $u$, первоначально является возбужденной, в то время как вторая компонента, $w$, первоначально находится в состоянии покоя. Тогда легко показать, что

$$
\begin{aligned}
& u=\frac{U}{\alpha_{1}+\left|\alpha_{2}\right|}\left[\left|\alpha_{2}\right| \cos \left(k x-\omega_{1} t\right)+\alpha_{1} \cos \left(k x-\omega_{2} t\right)\right], \\
& w=-\frac{2 \alpha_{1}\left|\alpha_{2}\right| U}{\alpha_{1}+\left|\alpha_{2}\right|} \sin \left(\gamma_{-} t\right) \sin \left(k x-\gamma_{+} t\right),
\end{aligned}
$$

где $\gamma_{ \pm}=\left(\omega_{2} \pm \omega_{1}\right) / 2$. Это решение описывает процесс обмена энергией между волнами, распространяюшимися в двух компонентах $u$ и $w$. 


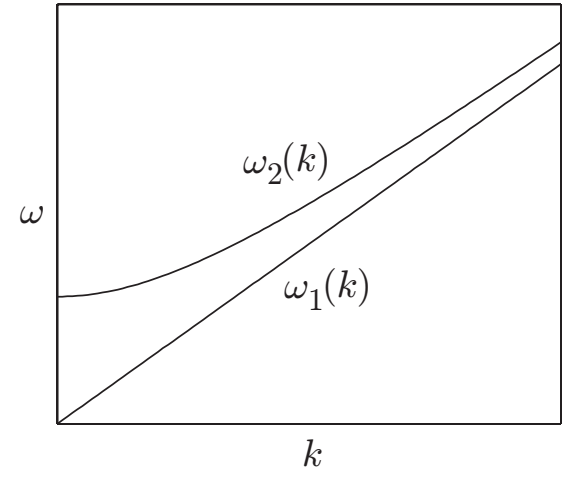

a

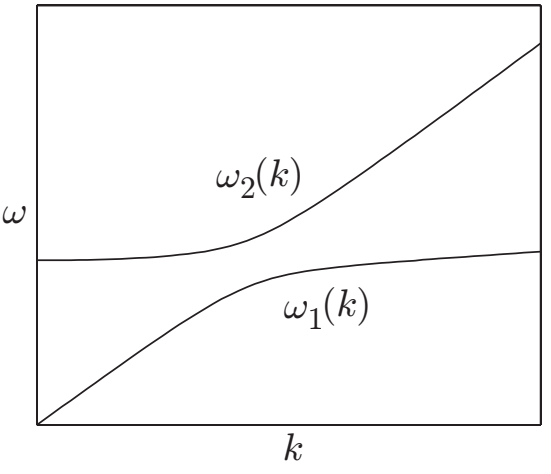

6

Рис. 1. Дисперсионная кривая для случаев $c=1$ (а) и $c \neq 1$ (б).

Полный обмен энергией между двумя компонентами, от нулевого до ненулевого значения, происходит, если выполнено условие

$$
\left(c^{2}-1\right) k^{2}=\delta^{2}-1 .
$$

В этом случае $\nu_{1}^{2}=\nu_{2}^{2}=\nu^{2}, \alpha_{1}=-\alpha_{2}=\delta^{-1}$, и решение (4) сводится к формулам

$$
\begin{aligned}
u & =U \cos \left(\gamma_{-} t\right) \cos \left(k x-\gamma_{+} t\right), \\
w & =-U \delta^{-1} \sin \left(\gamma_{-} t\right) \sin \left(k x-\gamma_{+} t\right) .
\end{aligned}
$$

Это решение описывает периодический обмен энергией с периодом $T=2 \pi / \gamma_{-}$между волнами, распространяющимися по компонентам $u$ и $w$. Этот процесс изображен на рис. 2 для $c=\delta=1, k=1.6$. Если $\nu^{2} \gg \delta$, то период $T$ обмена энергией стремится к бесконечности.

Если отношение $c=1$, то условие (5) может быть выполнено только в случае, если $\delta=1$ при произвольном параметре $k$. Если $c \neq 1$, то условие $(5)$ не выполняется ни при каком $k$, если $\left(\delta^{2}-1\right) /\left(c^{2}-1\right)<0$. В противном случае это условие будет выполняться только тогда, когда волновое число $k=\left(\left(\delta^{2}-1\right) /\left(c^{2}-1\right)\right)^{1 / 2}$.

Если параметр $\gamma_{-}=\left(\omega_{2}-\omega_{1}\right) / 2$ достаточно мал, т.е. частоты $\omega_{1}$ и $\omega_{2}$ достаточно близки при некотором волновом числе $k$, то компонента $u$ решения (6) оказывается почти в положении равновесия при $t=\pi / 2 \gamma_{-}, 3 \pi / 2 \gamma_{-}, \ldots$. При $t=0, \pi / \gamma_{-}, \ldots$ наблюдается обратная ситуация.

Частичный обмен энергией между двумя компонентами системы имеет место при $\left(c^{2}-1\right) k^{2} \neq\left(\delta^{2}-1\right)$. Тогда $\nu_{1}^{2} \neq \nu_{2}^{2}$, и компонента $u$ решения (4) может быть записана в виде

$$
u=\tilde{U}(t) \cos \left[k x-\omega_{2} t+\theta(t)\right]
$$

где

$$
\begin{gathered}
\widetilde{U}(t)=\frac{U}{\alpha_{1}+\left|\alpha_{2}\right|} \sqrt{\alpha_{1}^{2}+\alpha_{2}^{2}+2 \alpha_{1}\left|\alpha_{2}\right| \cos \left(\omega_{2}-\omega_{1}\right) t}, \\
\theta(t)=\operatorname{arctg}\left[\frac{\left|\alpha_{2}\right| \sin \left(\omega_{2}-\omega_{1}\right) t}{\alpha_{1}+\left|\alpha_{2}\right| \cos \left(\omega_{2}-\omega_{1}\right) t}\right] .
\end{gathered}
$$



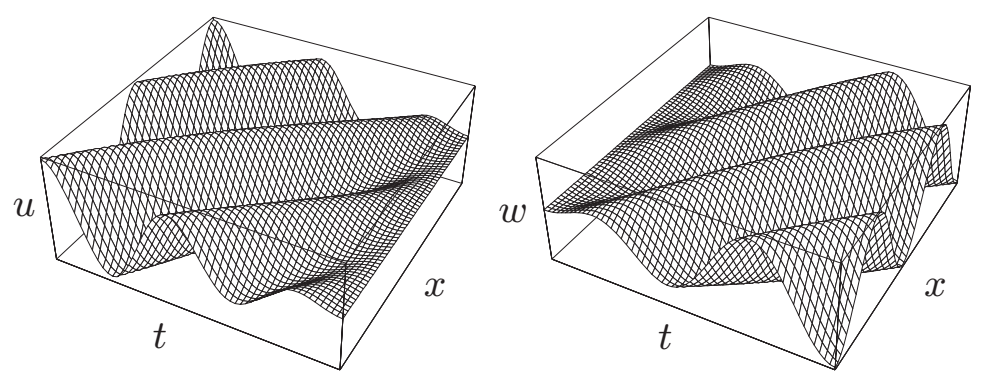

Рис. 2. Полный обмен энергией в линейном двухволновом решении (6).

При этом компонента $w$ остается такой же, как и в решении (4). Амплитуда волны, распространяюшейся в компоненте $u$, осциллирует в пределах между своим исходным значением $U$ и меньшим ненулевым значением $U\left|\alpha_{1}-\right| \alpha_{2}|| /\left(\alpha_{1}+\left|\alpha_{2}\right|\right)$.

Рассматривая суперпозицию всех четырех возможных волн с одним и тем же волновым числом $k$, можно найти решения для двух пар волн, распространяюшихся в противоположных направлениях и описываюших периодический обмен энергией между стоячими волнами в компонентах $u$ и $w$ (см. [13]). Однако в данной работе рассматриваются только решения для суперпозиции двух сонаправленных волн, обсуждавшиеся выше.

\section{3. АСИМПТОТИЧЕСКИЕ МОДЕЛИ СЛАБОНЕЛИНЕЙНЫХ РЕШЕНИЙ}

В случае $c=1$ точные нелинейные решения, описывающие обмен энергией между волнами, распространяюшимися в компонентах $u$ и $w$, могут быть построены в терминах эллиптических функций Якоби [13]. При этом, вводя новые переменные $p=u-w, q=$ $u+\delta^{2} w$, можно расшепить систему связанных уравнений в уравнение синус-Гордон для переменной $p$ и линейное волновое уравнение для переменной $q$.

В обшем случае можно изучать слабонелинейные решения, сводя связанные уравнения синус-Гордон (2) к связанным уравнениям, описывающим медленную пространственно-временную эволюцию волновых амплитуд. Медленная эволюция задается многомасштабным асимптотическим разложением

$$
\left(\begin{array}{l}
u \\
w
\end{array}\right)=\varepsilon\left(\begin{array}{l}
u_{1} \\
w_{1}
\end{array}\right)+\varepsilon^{2}\left(\begin{array}{c}
u_{2} \\
w_{2}
\end{array}\right)+\varepsilon^{3}\left(\begin{array}{l}
u_{3} \\
w_{3}
\end{array}\right)+O\left(\varepsilon^{4}\right),
$$

в котором в случае двух волн нужно положить

$$
\left(\begin{array}{c}
u_{1} \\
w_{1}
\end{array}\right)=A e^{i\left(k x-\omega_{1} t\right)}\left(\begin{array}{c}
1 \\
\alpha_{1}
\end{array}\right)+C e^{i\left(k x-\omega_{2} t\right)}\left(\begin{array}{c}
1 \\
\alpha_{2}
\end{array}\right)+\text { к.с. }
$$

Здесь $\varepsilon \ll 1$ представляет собой малый параметр, а величины $A$ и $C$ суть функции только медленных переменных.

Мы будем исследовать два различных режима, для каждого из которых предлагается своя асимптотическая модель. Мы получаем один или другой режим в зависимости от того, на каком пространственном масштабе, $\varepsilon^{-1}$ или $\varepsilon^{-2}$, происходит изменение амплитуд волн. Как будет показано ниже, дисперсионные члены оказываются сушественными в первом режиме, в то время как во втором режиме ими можно пренебречь. 
Для того чтобы получить первую модель, будем следовать подходу работы [14]. Предположим, что решения периодичны с пространственным периодом $\varepsilon^{-1} L$, и введем пространственную переменную больших расстояний $\chi=\varepsilon x$ и переменные медленных времен $\tau=\varepsilon t$ и $T=\varepsilon^{2} t$. Тогда в порядке $O(\varepsilon)$ (т.е. в главном порядке исследуемого разложения) получаются линейные уравнения для переменных $u_{1}$ и $w_{1}$, которые задают двухволновое решение (7) с $A=A(\tau, T, \chi)$ и $C=C(\tau, T, \chi)$. В порядке $O\left(\varepsilon^{2}\right)$ получаем два условия разрешимости $A_{\tau}+v_{g 1} A_{\chi}=0$ и $C_{\tau}+v_{g 2} C_{\chi}=0$. Вводя переменные

$$
\eta=\chi-v_{g 1} \tau, \quad \xi=\chi-v_{g 2} \tau
$$

и предполагая, что $v_{g 1} \neq v_{g 2}$, эти условия можно записать в виде

$$
A=A(\eta, T), \quad C=C(\xi, T) .
$$

Тогда члены порядка $O\left(\varepsilon^{2}\right)$ в разложении примут вид

$$
\left(\begin{array}{c}
u_{2} \\
w_{2}
\end{array}\right)=A_{2}(\eta, \xi, T) e^{i\left(k x-\omega_{1} t\right)}\left(\begin{array}{c}
1 \\
\alpha_{1}
\end{array}\right)+C_{2}(\eta, \xi, T) e^{i\left(k x-\omega_{2} t\right)}\left(\begin{array}{c}
1 \\
\alpha_{2}
\end{array}\right) .
$$

В порядке $O\left(\varepsilon^{3}\right)$ обычным образом получаются условия разрешимости:

$$
\begin{aligned}
& \left(\partial_{\tau}+v_{g 1} \partial_{\chi}\right) A_{2}+A_{T}=\frac{i}{2} \omega_{1}^{\prime \prime} A_{\chi \chi}+i \mu_{1}|A|^{2} A+i \mu_{2}|C|^{2} A, \\
& \left(\partial_{\tau}+v_{g 2} \partial_{\chi}\right) C_{2}+C_{T}=\frac{i}{2} \omega_{2}^{\prime \prime} C_{\chi \chi}+i \mu_{3}|C|^{2} C+i \mu_{4}|A|^{2} C,
\end{aligned}
$$

где

$$
\begin{aligned}
v_{g i} & =\omega_{i}^{\prime}=\frac{k}{\omega_{i}} \frac{1+\alpha_{i}^{2} \delta^{2} c^{2}}{1+\alpha_{i}^{2} \delta^{2}} \\
\omega_{i}^{\prime \prime} & =\frac{1-v_{g i}^{2}+\alpha_{i}^{2} \delta^{2}\left(c^{2}-v_{g i}^{2}\right)+4 \omega_{i}^{2} \alpha_{i}\left(v_{g i}-k / \omega_{i}\right)\left(v_{g i}-c^{2} k / \omega_{i}\right)}{\omega_{i}\left(1+\alpha_{i}^{2} \delta^{2}\right)} \\
\mu_{1} & =\frac{\delta^{2}\left(1-\alpha_{1}\right)^{4}}{4 \omega_{1}\left(1+\alpha_{1}^{2} \delta^{2}\right)}, \quad \mu_{2}=\frac{\delta^{2}\left(1-\alpha_{1}\right)^{2}\left(1-\alpha_{2}\right)^{2}}{2 \omega_{1}\left(1+\alpha_{1}^{2} \delta^{2}\right)} \\
\mu_{3} & =\frac{\delta^{2}\left(1-\alpha_{2}\right)^{4}}{4 \omega_{2}\left(1+\alpha_{2}^{2} \delta^{2}\right)}, \quad \mu_{4}=\frac{\delta^{2}\left(1-\alpha_{1}\right)^{2}\left(1-\alpha_{2}\right)^{2}}{2 \omega_{2}\left(1+\alpha_{2}^{2} \delta^{2}\right)}
\end{aligned}
$$

Записывая частные призводные в терминах переменных $\eta$ и $\xi$ из (8), получим

$$
\begin{aligned}
& \left(v_{g 1}-v_{g 2}\right) A_{2 \xi}+A_{T}=\frac{i}{2} \omega_{1}^{\prime \prime} A_{\eta \eta}+i \mu_{1}|A|^{2} A+i \mu_{2}|C|^{2} A, \\
& \left(v_{g 2}-v_{g 1}\right) C_{2 \eta}+C_{T}=\frac{i}{2} \omega_{2}^{\prime \prime} C_{\xi \xi}+i \mu_{3}|C|^{2} C+i \mu_{4}|A|^{2} C .
\end{aligned}
$$

Поскольку указанные решения пространственно-периодичны с периодом $L$ по переменной $\chi$, из формул (8) следует, что решения также должны быть периодичны по переменным $\eta$ и $\xi$ с тем же периодом $L$. Тогда можно проинтегрировать первое из двух уравнений, записанных выше, по переменной $\xi$ в пределах от 0 до $L$, учитывая, что $A=A(\eta, T)$, 
а затем проинтегрировать второе уравнение по переменной $\eta$ от 0 до $L$, учитывая, что $C=C(\xi, T)$. В результате получим

$$
\begin{aligned}
& A_{T}=\frac{i}{2} \omega_{1}^{\prime \prime} A_{\eta \eta}+i \mu_{1}|A|^{2} A+i \mu_{2}\left(\frac{1}{L} \int_{0}^{L}|C|^{2} d \xi\right) A, \\
& C_{T}=\frac{i}{2} \omega_{2}^{\prime \prime} C_{\xi \xi}+i \mu_{3}|C|^{2} C+i \mu_{4}\left(\frac{1}{L} \int_{0}^{L}|A|^{2} d \eta\right) C .
\end{aligned}
$$

Эти уравнения описывают слабонелинейную эволюцию волновых амплитуд в общем случае, когда $v_{g 1} \neq v_{g 2}$.

Вторую асимптотическую модель можно получить, вводя переменные большого расстояния $X=\varepsilon^{2} x$ и медленного времени $T=\varepsilon^{2} t$. В обшем нерезонансном случае с помощью длинных, но простых выкладок можно получить систему связанных уравнений на волновые амплитуды $A=A(T, X)$ и $C=C(T, X)$ (см. [13]), которая в главном порядке имеет вид

$$
\begin{aligned}
& i\left(A_{T}+v_{g 1} A_{X}\right)+\mu_{1}|A|^{2} A+\mu_{2}|C|^{2} A=0, \\
& i\left(C_{T}+v_{g 2} C_{X}\right)+\mu_{3}|C|^{2} C+\mu_{4}|A|^{2} C=0,
\end{aligned}
$$

а коэффициенты задаются, как и раньше, формулами (10).

Заметим, что асимптотические модели, аналогичные моделям (11) и (12), были недавно построены [15] для случая противоположно распространяющихся волн.

Обе вьшеприведенные асимптотические модели обладают одним и тем же решением в виде связанных волн (СВ-решением), не зависяшим от переменной $x$ :

$$
A=A_{0} e^{i \omega_{a} T}, \quad C=C_{0} e^{i \omega_{c} T}
$$

где $\omega_{a}=\mu_{1}\left|A_{0}\right|^{2}+\mu_{2}\left|C_{0}\right|^{2}$ и $\omega_{c}=\mu_{3}\left|C_{0}\right|^{2}+\mu_{4}\left|A_{0}\right|^{2}$. Подставляя решение (13) в анзац (7), получим решение в главном порядке:

$$
\left(\begin{array}{c}
u \\
w
\end{array}\right)=\varepsilon A_{0} e^{i\left(k x-\omega_{1} t+\omega_{a} \varepsilon^{2} t\right)}\left(\begin{array}{c}
1 \\
\alpha_{1}
\end{array}\right)+\varepsilon C_{0} e^{i\left(k x-\omega_{2} t+\omega_{c} \varepsilon^{2} t\right)}\left(\begin{array}{c}
1 \\
\alpha_{2}
\end{array}\right)+\text { к.c. }
$$

В пределе $\varepsilon \rightarrow 0$ решение (14) преврашается в линейное решение (4), если

$$
\varepsilon A_{0}=\frac{\left|\alpha_{2}\right| U}{2\left(\alpha_{1}+\left|\alpha_{2}\right|\right)}, \quad \varepsilon C_{0}=\frac{\alpha_{1} U}{2\left(\alpha_{1}+\left|\alpha_{2}\right|\right)}
$$

В случае $\varepsilon \neq 0$ решение (14) задает обобщение линейных решений ввиду наличия в нем поправок порядка $O\left(\varepsilon^{2}\right)$ к частотам $\omega_{1,2}(k)$, индуцированных нелинейностью.

Рассмотрим теперь более подробно каждую из указанных асимптотических моделей. 


\section{4. БЕЗДИСПЕРСИОННОЕ ПРИБЛИЖЕНИЕ}

Бездисперсионная модель (12) оказывается интегрируемой в квадратурах. В самом деле, легко проверить, что величины $A A^{*}$ и $C C^{*}$ сохраняются:

$$
\left(A A^{*}\right)_{T}+v_{g 1}\left(A A^{*}\right)_{X}=0, \quad\left(C C^{*}\right)_{T}+v_{g 2}\left(C C^{*}\right)_{X}=0
$$

что приводит к тому, что общее решение системы (12) имеет вид

$$
A=a\left(\eta_{1}\right) e^{i \phi}, \quad C=c\left(\xi_{1}\right) e^{i \psi},
$$

где

$$
\begin{aligned}
\phi_{T}+v_{g 1} \phi_{X} & =\mu_{1} a^{2}\left(\eta_{1}\right)+\mu_{2} c^{2}\left(\xi_{1}\right), \\
\psi_{T}+v_{g 2} \psi_{X} & =\mu_{3} c^{2}\left(\xi_{1}\right)+\mu_{4} a^{2}\left(\eta_{1}\right),
\end{aligned}
$$

а $a\left(\eta_{1}\right)$ и $c\left(\xi_{1}\right)$ суть произвольные функции своих аргументов $\eta_{1}=X-v_{g 1} T$ и $\xi_{1}=X-$ $v_{g 2} T$.

Обшее решение системы (17) может быть записано в виде

$$
\begin{aligned}
& \phi=F\left(\eta_{1}\right)+\mu_{1} T a^{2}\left(\eta_{1}\right)+\frac{\mu_{2}}{v_{g 1}-v_{g 2}} \int_{0}^{\xi_{1}} c^{2}(\mu) d \mu, \\
& \psi=G\left(\xi_{1}\right)+\mu_{3} T c^{2}\left(\xi_{1}\right)+\frac{\mu_{4}}{v_{g 2}-v_{g 1}} \int_{0}^{\eta_{1}} a^{2}(\mu) d \mu,
\end{aligned}
$$

где $F\left(\eta_{1}\right)$ и $G\left(\xi_{1}\right)$ суть произвольные функции своих аргументов. CВ-решение, обсуждавшееся в предыдушем разделе, представляет собой частное решение, отвечаюшее начальным данным

$$
\begin{aligned}
\left.a\right|_{T=0} & =A_{0},\left.\quad c\right|_{T=0}=C_{0}, \\
\left.\phi\right|_{T=0} & =0,\left.\quad \psi\right|_{T=0}=0 .
\end{aligned}
$$

Из вида общего решения (16), (18) следует, что всякое малое возмушение условий (20) вызывает лишь малое возмушение фаз CB-решения. Хотя всякое малое возмушение условий (19) сушественно влияет на фазы СВ-решения, оно приводит только лишь к малым возмушениям соответствуюших амплитуд. Поэтому в данном режиме модуляционная неустойчивость отсутствует.

\section{5. МОДУЛЯЦИОННАЯ НЕУСТОЙЧИВОСТЬ}

Модуляционная неустойчивость слабонелинейных решений (14) может быть тем не менее исследована с использованием уравнений (11), которые оказываются справедливыми для возмущений с пространственным масштабом $O\left(\varepsilon^{-1}\right)$.

Перед тем как рассмотреть обший случай, обратимся к случаю $c=1$. Тогда имеем $\mu_{1}=\mu_{2}=\mu_{4}=0, \omega_{1}^{\prime \prime}=0$, и уравнения (11) принимают вид

$$
A_{T}=0, \quad C_{T}=\frac{i \omega_{2}^{\prime \prime}}{2} C_{\xi \xi}+i \mu_{3}|C|^{2} C,
$$


где $A=A(\eta, T)$ и $C=C(\xi, T)$, а коэффишиенты уравнения задаются условиями $\omega_{1}=k$ и $\omega_{2}=\sqrt{\delta^{2}+1+k^{2}}$. При этом на функцию $A$ получается тривиальное линейное уравнение, а функция $C$ описывается стандартным нелинейным уравнением Шредингера, что напоминает о возможности расщепить уравнения синус-Гордон в данном случае.

Рассмотрим некоторое обобщение вышеприведенной ситуации, а именно случай, когда параметр $c$ близок к единице, т.е. $c^{2}=1+\rho$, где $|\rho| \ll 1$. Этот случай важен с точки зрения возможных приложений, и он может быть исследован с использованием асимптотического анализа по переменной $\rho$. Можно показать, что с точностью до поправок первого порядка по переменной $\rho$ поведение системы по-прежнему описывается уравнениями (21), в которых коэффициенты следует вычислить, используя следующие приближения:

$$
\begin{aligned}
& \omega_{1}=k+\frac{1}{2} \rho k \frac{\delta^{2}}{\delta^{2}+1}+O\left(\rho^{2}\right) \\
& \omega_{2}=\sqrt{\delta^{2}+1+k^{2}}+\frac{1}{2} \rho k^{2} \frac{1}{\left(\delta^{2}+1\right) \sqrt{\delta^{2}+1+k^{2}}}+O\left(\rho^{2}\right) .
\end{aligned}
$$

В самом деле, в этом приближении легко проверить, что

$$
\alpha_{1}=1-\rho k^{2} /\left(\delta^{2}+1\right)+O\left(\rho^{2}\right), \quad \alpha_{2}=-\delta^{-2}\left(1+\rho k^{2} /\left(\delta^{2}+1\right)\right)+O\left(\rho^{2}\right),
$$

откуда, предполагая $k \sim \delta \sim 1$, можно вывести асимптотические условия $\mu_{1} \sim O\left(\rho^{4}\right)$, $\mu_{2} \sim \mu_{4} \sim O\left(\rho^{2}\right)$ и $\mu_{3} \sim O(1)$. Поскольку оба параметра $\omega_{2}^{\prime \prime}$ и $\mu_{3}$ положительны, приходим к заключению, что в случае, когда $c$ близко к единице, CВ-решение оказывается модуляционно неустойчивым.

В общем случае решения уравнений (11) ищутся в виде

$$
\begin{aligned}
& A(\eta, T)=A_{0}(T)\left(1+a_{1} e^{i J \eta+S_{a} T}+a_{2}^{*} e^{-i J \eta+S_{a}^{*} T}\right), \\
& C(\xi, T)=C_{0}(T)\left(1+c_{1} e^{i J \xi+S_{c} T}+c_{2}^{*} e^{-i J \xi+S_{c}^{*} T}\right),
\end{aligned}
$$

который отвечает синусоидальным возмущениям CВ-решения $A_{0}(T), C_{0}(T)$, задаваемого выражением (14). Выбор волнового числа $J$ возмушения ограничивается условием периодичности по $x$. Пренебрегая членами второго порядка по амплитуде возмушения, из (11) получим две независимые линейные системы с дисперсионными соотношениями

$$
S_{a}^{2}=\mu_{1}\left|A_{0}\right|^{2} \omega_{1}^{\prime \prime} J^{2}-\frac{\omega_{1}^{\prime \prime 2} J^{4}}{4}, \quad S_{c}^{2}=\mu_{3}\left|C_{0}\right|^{2} \omega_{2}^{\prime \prime} J^{2}-\frac{\omega_{2}^{\prime \prime 2} J^{4}}{4}
$$

С помощью выражения для $\omega_{2}^{\prime \prime}(10)$ можно показать, что величина $\omega_{2}^{\prime \prime}$ всегда положительна. Тогда, поскольку $\mu_{3}>0$, получим, что уравнение на величину $S_{c}$ имеет корень с положительной вешественной составляющей при достаточно малых $J$, что соответствует неустойчивой моде. Из формулы (3) следует, что величина $\omega_{1}^{\prime \prime}$ отрицательна при мальх $k$, положительна при больших $k$ и стремится к нулю при $k \rightarrow \infty$. Поскольку $\mu_{1}>0$, величина $S_{a}$ чисто мнимая при малых $k$, а потому неустойчивая мода не возникает, в то время как при бо́льших $k$, при которых $\omega_{1}^{\prime \prime}>0$, есть неустойчивая мода.

Ниже мы будем предполагать, что либо величина $k$ такова, что $\omega_{1}^{\prime \prime}<0$, либо неустойчивость в компоненте $C$ оказывается доминируюшей, что имеет место в численном 
эксперименте, обсуждаемом в следующем разделе. Тогда, используя условия (15) на величину $\left|C_{0}\right|$ и возвращаясь к нерастянутым переменным с $s_{c}=\varepsilon^{2} S_{c}$ и $j=\varepsilon J$, получим, что дисперсионное соотношение для возмущений решения (14), описывающих обмен энергией, имеет вид

$$
s_{c}^{2}=\frac{\mu_{3} \omega_{2}^{\prime \prime} \alpha_{1}^{2} U^{2} j^{2}}{4\left(\alpha_{1}+\left|\alpha_{2}\right|\right)^{2}}-\frac{\omega_{2}^{\prime \prime 2} j^{4}}{4}
$$

где следует положить $U=O(\varepsilon)$ и $j \sim \varepsilon^{-1}$, для того чтобы обеспечить формальную справедливость разложения. Если только позволяет геометрия задачи, то наиболее неустойчивая мода с инкрементом нарастания $s_{*}$ имеет место при волновом числе $j_{*}$, где

$$
s_{*}=\frac{\mu_{3} \alpha_{1}^{2} U^{2}}{4\left(\alpha_{1}+\left|\alpha_{2}\right|\right)^{2}}, \quad j_{*}^{2}=\frac{\mu_{3} \alpha_{1}^{2} U^{2}}{2 \omega_{2}^{\prime \prime}\left(\alpha_{1}+\left|\alpha_{2}\right|\right)^{2}} .
$$

\section{6. ЧИСЛЕННЫЙ ЭКСПЕРИМЕНТ}

Представим теперь результаты наших численных экспериментов для системы (2), представляющей собой исходную систему связанных уравнений синус-Гордон. Это позволит оценить точность и применимость теоретических предсказаний для неустойчивости, представленных в разделе 5. Хотя в основном будут проверяться оценки инкремента нарастания и длины волны для модуляционной неустойчивости частных решений, обсуждавшихся в разделах 2 и 3 , мы также обсудим структуру решений в режиме, когда неустойчивости достигают максимума амплитуды.

Поскольку моделируются пространственно-периодические решения, естественно использовать базис Фурье по переменной $x$ для величин $u$ и $w$. Для шага по времени используется схема расщеплений вида $\hat{L}(\Delta t / 2) \hat{N}(\Delta t) \hat{L}(\Delta t / 2)$. Здесь разностный оператор $\hat{L}$ точно решает линейную часть системы, а оператор $\widehat{N}$ решает нелинейную часть системы с использованием метода Рунге-Кутта второго порядка, в которой нелинейные составляющие вычисляются псевдоспектрально. В целом схема представляет собой схему второго порядка по времени и имеет то преимушество, что она является точной при отсутствии нелинейных членов.

В проделанных вычислениях шаг по времени выбирался, как правило, в промежутке от 0.025 до 0.1 и в направлении $x$ аппроксимация проводилась по 256 точкам. Закон сохранения энергии выполняется в нелинейной схеме с большой точностью - типичная ошибка имеет порядок $10^{-5} \%$. Схема была протестирована на примере вычисления инкремента нарастания неустойчивости Бенджамина-Фейера при $c=1$ и $\varepsilon \ll 1$. Вычисленные значения инкремента нарастания, как правило, совпадали с хорошо известными теоретическими предсказаниями (см., например, [16]) с точностью до четырех значащих цифр.

Каждый численный эксперимент начинался с СВ-решения (4) при некоторых выбранных значениях параметров $k$ и $U$. Ширина области $2 \pi L_{c}$ при этом ограничена необходимостью выбора $L_{c}=n / k$, где $n$ - положительное целое число. Неустойчивость инициировалась с помошью фонового шума, который для величин $u$ и $w$ имел порядок, по крайней мере в тысячу раз меньший, чем величина $U$. Если нестабильность имеет место, то в эволюции системы наблюдается этап экспоненциального роста амплитуды 

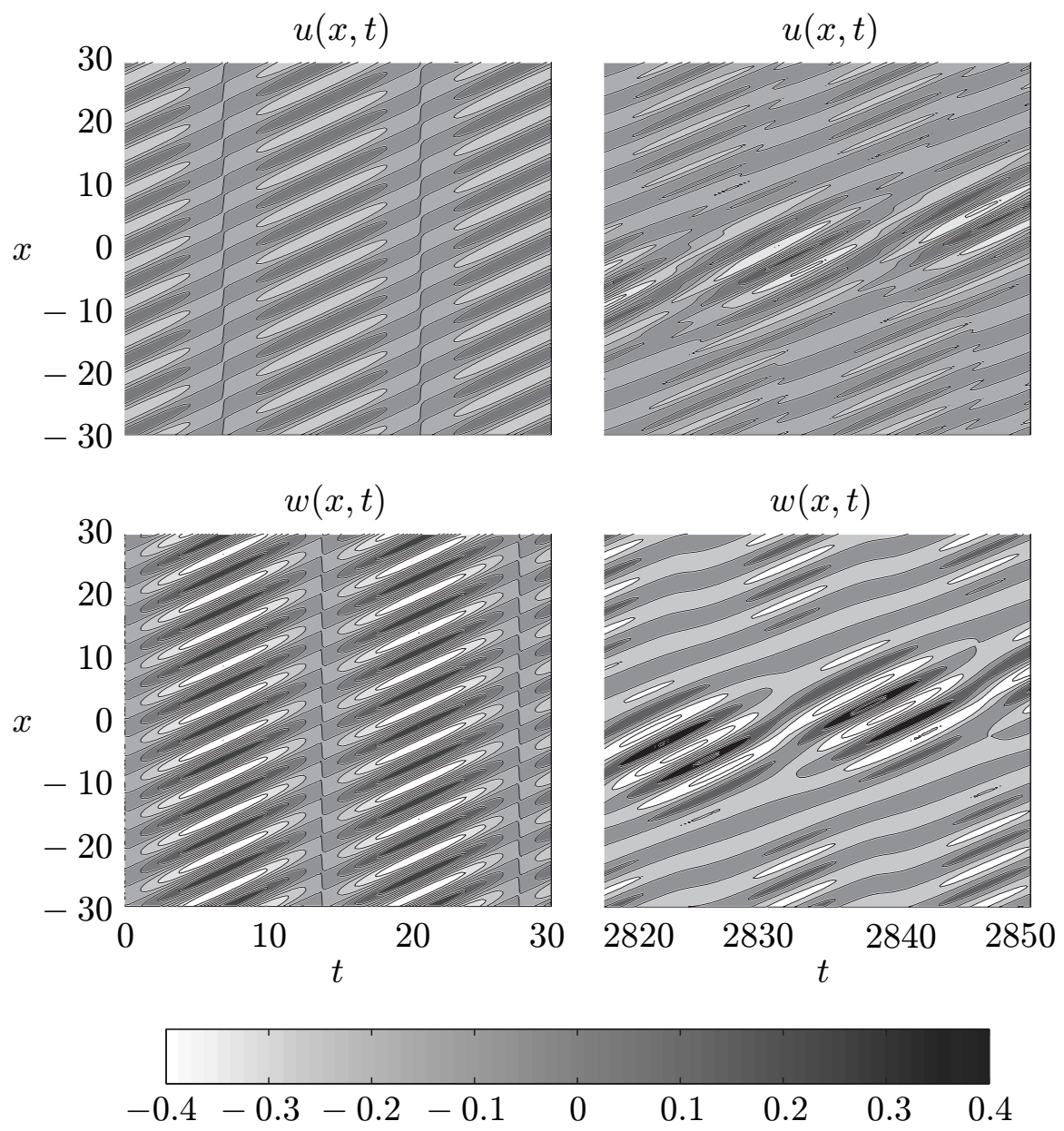

Рис. 3. Обмен энергией при $c=0.5, \delta=0.5, k=1$ и $U=0.2$.

Сравнение численно определенного инкремента нарастания $s_{n}$ с теоретически предсказанным значением $s_{c}$

\begin{tabular}{|c|c|c|c|c|c|c|}
\hline $\mathrm{c}$ & $\delta$ & $k$ & $U$ & $j$ & $s_{n}$ & $s_{c}$ \\
\hline 1 & 1 & 2 & 0.5 & $2 / 5$ & 0.01257 & 0.01262 \\
0.8 & 1 & 4 & 0.5 & $2 / 3$ & 0.006267 & 0.006454 \\
1.01 & 1 & 2 & 0.2 & $1 / 6$ & 0.001948 & 0.001950 \\
0.5 & 2 & 2 & 0.2 & $1 / 5$ & 0.004588 & 0.004592 \\
\hline
\end{tabular}

возмушения, инкремент нарастания которого может быть вычислен. Выборка численных результатов представлена в таблице, где параметр $j$ обозначает волновое число наблюдаемой неустойчивости (ограниченное размером области, в которой производятся 


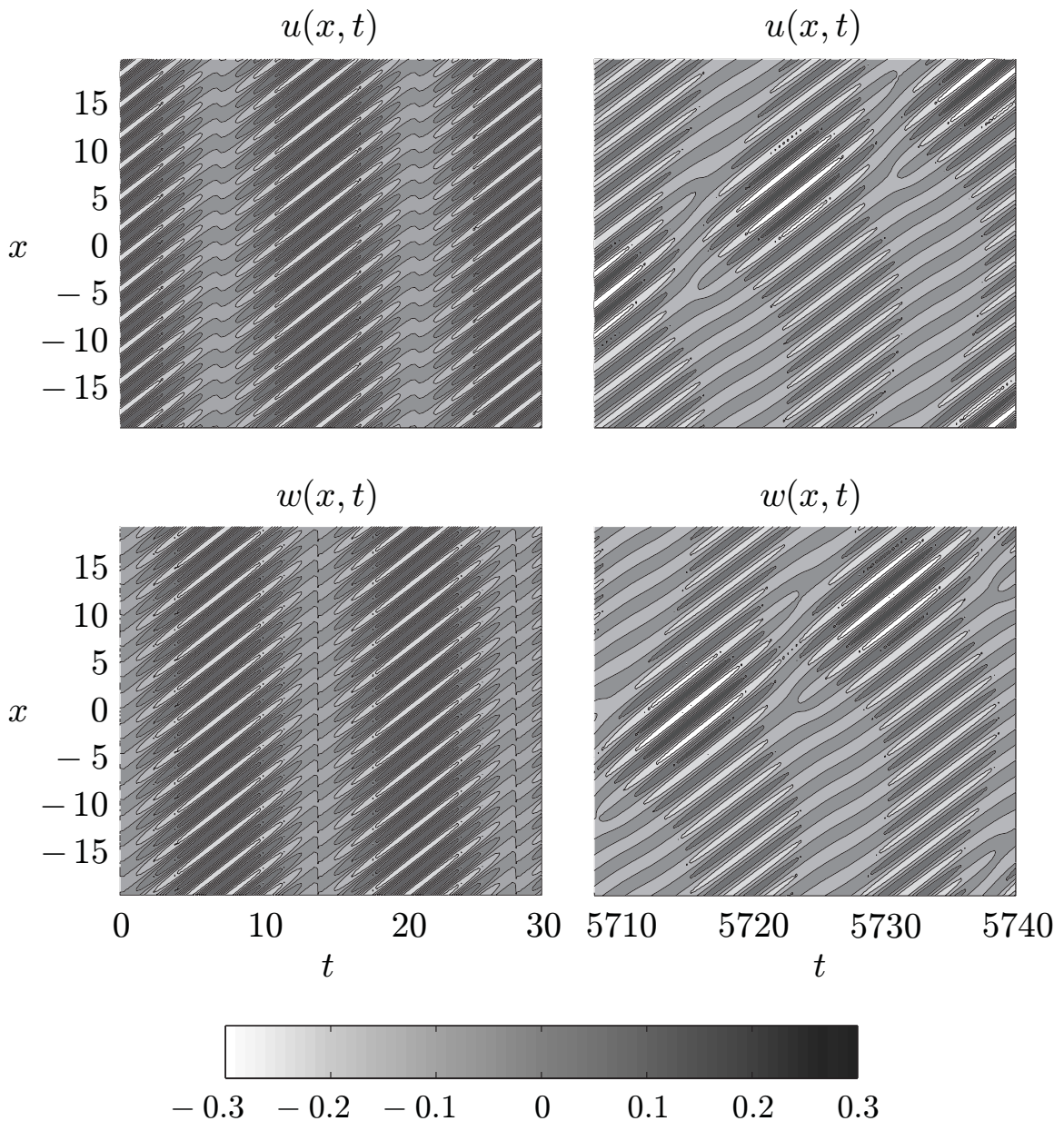

Рис. 4. Обмен энергией при $c=1.01, \delta=1, k=2$ и $U=0.2$.

вычисления), а параметр $s_{c}$ представляет собой теоретически предсказанный на основе формулы (22) инкремент нарастания. Приведенные результаты свидетельствуют о том, что формула (22) правильно предсказывает величину инкремента нарастания неустойчивых возмушений в широком диапазоне значений параметров $c, \delta, k$ и $j$ и вплоть до неожиданно больших значений амплитуды $U$.

Результаты численного эксперимента при $c=\delta=0.5, k=1$ и $U=0.2$ представлены на рис. 3. СВ-решение показано слева, а нелинейное решение в области, где амплитуда возмущения достигает максимума, изображено справа. Ширина области составляет $20 \pi$. Неустойчивость растет с волновым числом 0.1 , и длина волны неустойчивого возмущения тем самым совпадает с размером области. Также на рисунке можно видеть структуру первоначального обмена энергией в СВ-решении и то, как форма этого обмена видоизменяется в режиме, когда амплитуда возмушения достигает своего максимума. В последнем случае наибольшие отклонения от равновесия пульсируют между $u$ и $w$, и видно, что обмен энергией сохраняется и в нелинейном режиме. 
Соответствуюшие результаты вычислений при $c=1.01, \delta=1, k=2$ и $U=0.2$ в области шириной $12 \pi$ показаны на рис. 4 .

\section{7. ЗАКЛЮЧЕНИЕ}

В данной работе на примере двухкомпонентной системы связанных уравнений синус-Гордон были исследованы частные процессы обмена энергией между двумя физическими компонентами системы и влияние модуляционной неустойчивости на эти процессы. Было показано как теоретически, так и прямыми численными расчетами, что периодический процесс обмена энергией меж ду волнами, бегущими по этим двум компонентам, оказывается модуляционно неустойчивым, что приводит к формированию локализованных структур, но тем не менее не разрушает картину энергетического обмена между физическими компонентами системы.

Благодарности. Авторы благодарят В. А. Байкова, К. Мартеля, А. В. Михайлова и Д. Е. Пелиновского за полезные обсуждения. Авторы признательны за поддержку Математическому факультету Университета г. Лавборо.

\section{Список литературы}

[1] И. ШІ. Ахатов, В. А. Байков, К. Р. Хуснутдинова. Прикл. мат. мех. 1995. Т. 59. № 3. C. $376-384$.

[2] S. Yomosa. Phys. Rev. A. 1983. V. 27. P. 2120-2125.

[3] L. V. Yakushevich. Nonlinear Physics of DNA. Chichester: Wiley, 1998.

[4] А. В. Жибер, Н. Х. Ибрагимов, А. Б. Шабат. ДАН СССР. 1979. Т. 247. С. 1103-1105.

[5] S. Lie. Arch. Math. Naturv. 1881. V. 6. P. 112-124.

[6] N. H. Ibragimov (ed.). CRC Handbook of Lie Group Analysis of Differential Equations. V. 1. Symmetries, Exact Solutions, and Conservation Laws. Boca Raton: CRC, 1994.

[7] А. В. Жибер, А. Б. Шабат. ДАН СССР. 1979. Т. 247. С. 1103.

[8] Т. А. Конторова, Я. И. Френкель. ЖЭТФ. 1938. Т. 8. С. 89-95; С. 1340-1368.

[9] O. M. Braun, Yu. S. Kivshar. Phys. Rep. 1998. V. 306. P. 1-108.

[10] K. R. Khusnutdinova, V. V. Silberschmidt. Proc. Estonian Acad. Sci. Phys. Math. 2003. V. 52. P. $63-75$.

[11] Л. И. Мандельштам. Лекции по теории колебаний. М.: Наука, 1972

[12] В. И. Арнольд. Математические методы классической механики. М.: Наука, 1989.

[13] K. R. Khusnutdinova, D. E. Pelinovsky. Wave Motion. 2003. V. 38. P. 1-10.

[14] E. Knobloch, J. De Luca. Nonlinearity. 1990. V. 3. P. 975-980.

[15] C. Martel, E. Knobloch, J. M. Vega. Phys. D. 2000. V. 137. P. 94-123; C. Martel, J. M. Vega, E. Knobloch. Phys. D. 2003. V. 174. P. 198-217.

[16] C. Godrèche, P. Manneville (eds.). Hydrodynamics and Nonlinear Instabilities. Cambridge: Cambridge Univ. Press, 1998. 